\title{
MEASURING GALAXY BIAS FROM THE HIGH-ORDER CORRELATION FUNCTIONS
}

\author{
Y.P. JING \\ Research Center for the Early Universe, University of Tokyo \\ Bunkyo-ku, Tokyo 113, Japan
}

\begin{abstract}
In this talk, I will show how to determine the biasing factor $b$ from the high-order moments of galaxies. The determination is based on the analytical modeling of primordial peaks and virialized halos and is independent of the currently unknown density parameter $\Omega_{0}$ and other cosmological parameters. The observed high-oder moments of the APM galaxies require that the biasing factor $b$ be very close to 1 , i.e. the optical galaxies are an unbiased tracer of the underlying mass distribution (on quasilinear scale). The theoretical argument can be easily generalized to the three-point correlation function and the bispectrum both of which can used as further observational tests to the important conclusion of $b \approx 1$ drawn from the high-order moments. Finally I present our preliminary results of the three-point correlation functions for the Las Campanas Redshift Survey.
\end{abstract}

\section{Introduction}

A fundamental problem in cosmology is to understand how the spatial distribution of galaxies (and of galaxy clusters) is related to that of the underlying mass. The possibility for a galaxy bias (i.e. galaxies do not faithfully trace the underlying matter in spatial distribution) is advocated both by observations and by theories. It is therefore very important to determine the relation between the distribution of galaxies and that of dark matter (DM). The distribution of DM cannot be directly measured by telescopes because of its very nature. People attempted to understand the DM distribution by studying the dynamics of galaxies since the peculiar motions of galaxies are closely related to the distribution of DM. Unfortunately, these studies can only yield a combination of $\Omega_{0}^{0.6} \sigma_{8}$ where $\Omega_{0}$ is the den- 
sity parameter of the Universe and $\sigma_{8}$ is the rms tophat density contrast of radius $8 h^{-1} \mathrm{Mpc}$. Moreover, there are considerable discrepancies in the $\Omega_{0}^{0.6} \sigma_{8}$ value among different measurements. In this talk, I will show how to determine the biasing factor $b$ from the high-order moments of galaxies, which is based on the analytical modeling of primordial peaks and virialized halos and is independent of the currently unknown density parameter $\Omega_{0}$ and other cosmological parameters. The method is distinct from the related work of Gaztanaga \& Frieman (1994) which is based on a simple non-physical parameterization of the bias relation.

\section{Measuring the biasing factor $b$}

The peak model and the halo model are generally used to prescribe the formation of galaxies. Recently, we have analytically derived the high-order moments for the primordial peaks and virialized halos (Mo, Jing, \& White 1997 ) based on the method of Mo \& White (1996). The high order moments of the peaks or the halos, e.g. the skewness $S_{3}(R)$, the kurtosis $S_{4}(R)$ and $S_{5}(R)$, are functions of the biasing factor $b$, the shape of the linear power spectrum, and the formation time $z_{f}$ only. Our analytical model has been tested against a set of high-resolution cosmological N-body simulations, with a conclusion that the model works very well in the quasilinear regime.

In Figure 1, I present our predictions for the high-order moments $S_{j, g}$ $(j=3,4,5)$ of peaks and halos as a function of the linear bias parameter $b$. In this plot, a CDM-like power spectrum with $\Gamma=0.2$ is assumed. This spectrum is known to be consistent with the angular correlation function of the APM galaxies (Efstathiou, Sutherland \& Maddox 1990). The currently best estimates of the high-order moments for optical galaxies are those of Gatzanaga (1994) based on the APM survey, which are plotted on the figure with error-bars. Recent observations of galaxies at high redshift indicate that bulk of galaxies is formed at $z_{f} \gtrsim 1$. Figures 1 then implies that the optical galaxies are little biased relative to the distribution of underlying mass (i.e. $b \approx 1$ ). It is interesting to note that the observations of $S_{3}, S_{4}$ and $S_{5}$ for the APM galaxies are all consistent with the analytical model if $b \approx 1$.

A comparison with the observed moments of galaxy clusters is also interesting. Clusters of galaxies are generally believed to be halos formed recently $\left(z_{f}=0\right)$ and have a bias factor $b \approx 3 \sim 5$. Our model predicts the skewness $S_{3, h} \approx 2$ and the kurtosis $S_{4, h} \approx 8$ for such halos which are well consistent with the observations for the Abell clusters (Jing \& Zhang 1989) and for the APM clusters (Gatzanaga, Croft \& Dalton 1995).

\section{Further observational tests: the three-point correlation func-}




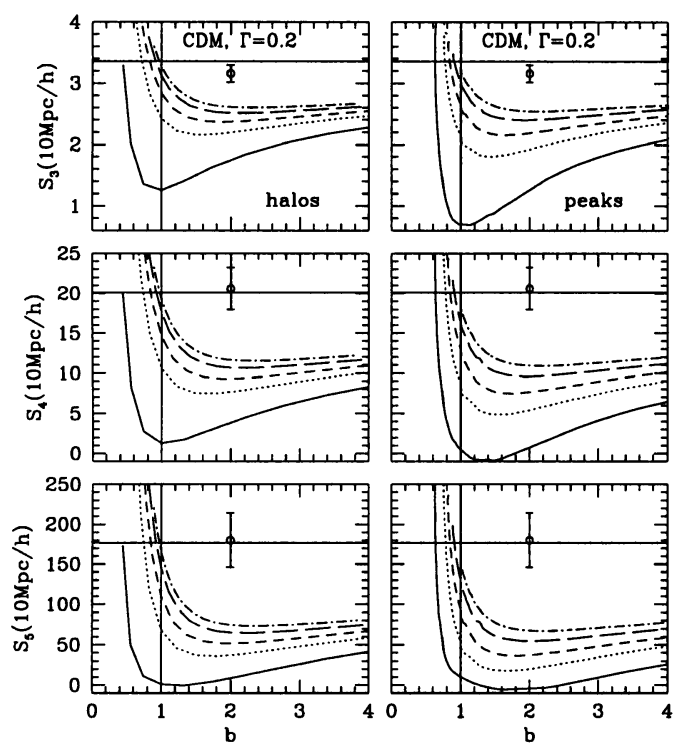

Figure 1. Model predictions for the high order moments $S_{j, g}(j=3,4$ and 5$)$ of halos (left panels) and peaks (right panels) at a radius $r=10 h^{-1} \mathrm{Mpc}$ as a function of the linear bias parameter $b$. Each curve shows result for a given formation time $z_{f}=0,0.5$, 1,2 and 4 (curves from bottom up). The horizontal lines show the values of $S_{j}$ for the mass density field calculated from quasilinear theory, whereas the data points (plotted arbitrarily at $b=2$ ) show the observational results for APM galaxies (Gaztanaga 1994)

\section{tion and the bispectrum}

With a simple extension of the argument of Mo et al. (1997), we can derive the bispectrum and the three-point correlation function for the DM halos and the primordial peaks. In this extension we assume the bias relation by derived Mo et al. (1997) holds at all scales. We have tested this extension against a set of high-resolution N-body simulations with $1.7 \times 10^{7}$ particles, and have found that the extension is very successful (Jing 1997, in preparation). Figure 2 illustrates such a test, where we compare the normalized bispectrum of $\mathrm{N}$-body dark matter halos (symbols) with the predictions of the extension. The extension predicts that the optical galaxies should closely follow the underlying dark matter in the bispectrum and in the three-point correlation function, if the biasing factor of the galaxies is about 1 as the high-order moments indicate $(\S 2)$ and the bulk of the galaxies is formed before redshift 1 . Both the amplitude and the shape dependence of the three-point correlation function and the bispectrum are potentially useful for constraining the bias factor and the linear power spectrum of the cosmological models (Fry 1994; Jing \& Börner 1997; Matsubara \& Suto 


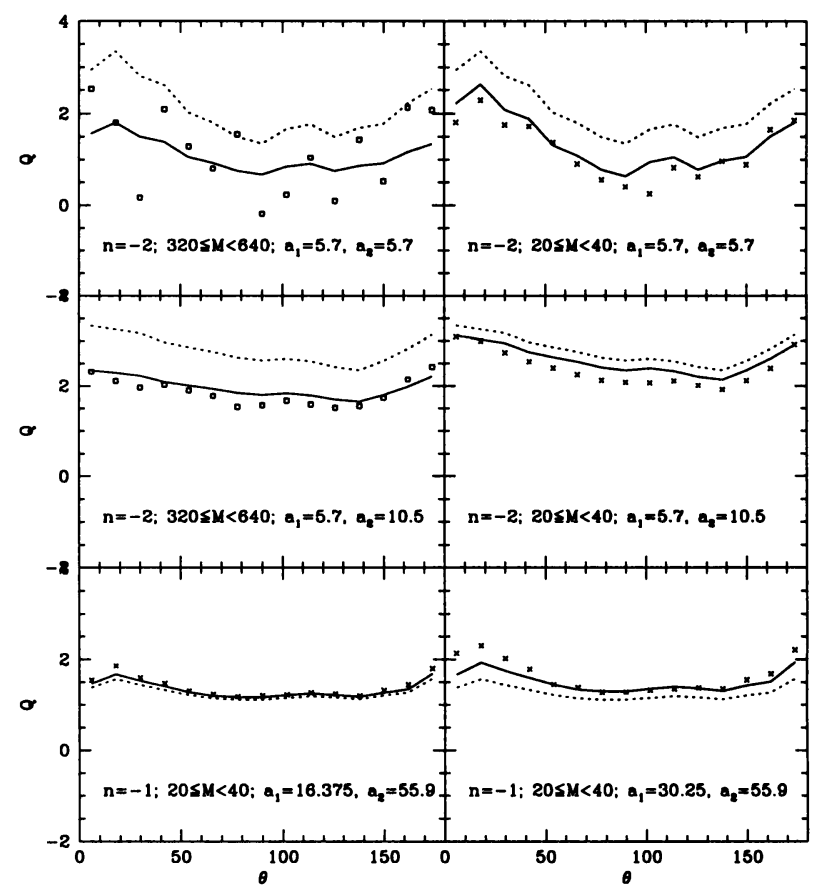

Figure 2. The normalized bispectrum $Q\left(k_{1}, k_{2}, \theta\right)$ of virliazed halos estimated from the scale-free simulations with the power spectrum index $n$ and $256^{3}$ particles (symbols), compared with the analytical predictions discussed in the text. The halos of mass $M$ (in unit of the particle mass) are formed at the scale factor $a_{1}$ and the clustering analysis is made at $a_{2}$.

1994; Matarrese et al. 1997).

\section{Preliminary results from the LCRS survey}

Here I present the preliminary results of our determination (Jing \& Börner 1997) of the three-point correlation function for the Las Campanas Redshift Survey. With this large sample we are able to determine the three-point correlation function of galaxies much more accurately than any previous works (e.g. Efstathiou \& Jedrzejewski 1984). The three-point correlation function is not only a powerful test to the bias problem as described above but also a generally important statistic to describe spatial distributions and dynamics of galaxies (Peebles 1980; Suto 1993).

We analysed two types of three-point correlations: the redshift space three-point correlation function $\zeta(s, u, v)$ and the projected three-point correlation function $\Pi\left(r_{p}, u, v\right)$. Our results are presented in Figure 3. The figure shows that the redshift three-point correlation function is approxi- 

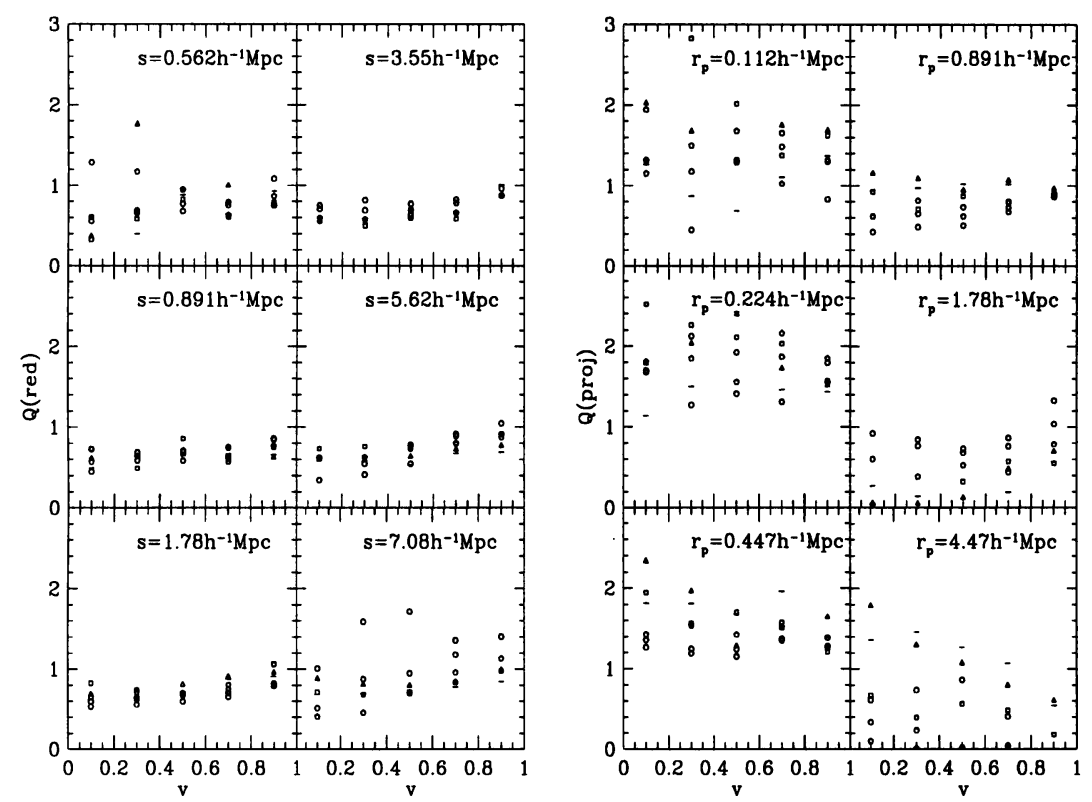

Figure 3. The normalized three-point correlation function of galaxies in the Las Campanas Redshift Survey. The left panel shows the result in the redshift space and the right panel shows the projected one. The $r, u, v$ convention of Peebles (1980) is adopted in the plot, and different symbols are for different values of $u$. More detailed account of the figure will be given in Jing \& Börner (1997)

mately hierarchical with the amplitude $Q($ red $) \approx 0.6$ (see e.g. Jing \& Börner 1997 for its definition). There is a tendency that $Q$ increases with $v$ especially for large triangles with $s>5 h^{-1} \mathrm{Mpc}$, qualitatively in agreement with the model predictions for the mass three-point correlation function (Jing \& Börner 1997). Similar properties are found for the projected one. However the projected $Q$ (proj) is about 1.5 for very small scales $r_{p} \leq 0.5 h^{-1} \mathrm{Mpc}$ and decreases with $r_{p}$ for larger $r_{p}$. If the three-point correlation function in real space is hierarchical, the projected one should also be hierarchical with $Q($ proj $)=Q$. Our results then support that the three-point correlation function on small scales is hierarchical with $Q \approx 1.5$, consistent with the conclusions of Peebles (1980) based on 2-D angular catalogues. The hierarchical form however seems not to hold for scales much larger than $0.5 h^{-1} \mathrm{Mpc}$ because $Q$ (proj) decreases with $r_{p}$ and increase with $v$ for $r_{p}>0.5 h^{-1} \mathrm{Mpc}$. A quantitative comparison between the observations and the CDM models is in progress, which will take account of the redshift distortion effect and the projection effect (Jing \& Börner 1997). 


\section{Conclusions}

In this talk I have presented our analytical model for calculating the highorder moments of peaks and halos in the quasilinear regime. With this model, the observed skewness and kurtosis of the Abell and the APM clusters are interpreted as a consequence of these clusters being recently formed massive halos. The high-oder moments of the APM galaxies are consistent with the model predictions if the galaxies are an unbiased tracer of the underlying mass distribution (on quasilinear scale). This model can even be extended to the normal three-point correlation function and the bispectrum (cf. Catelan et al. 1997), and thus our important conclusion that $b \approx 1$ can be further tested with these functions. Finally I presented the preliminary results of our determination of the three-point correlation functions for galaxies in the Las Campanas Redshift Survey.

\section{Acknowledgements}

The author gratefully acknowledges the postdoctoral fellowship from Japan Society for the Promotion of Science. Part of numerical computations were carried out on VPP300/16R and VX/4R at the Astronomical Data Analysis Center of the National Astronomical Observatory, Japan,

\section{References}

Catelan. P., Lucchin, F., Matarrese, S., Porciani, C. 1997, MNRAS (submitted); astro$\mathrm{ph} / 9708067$

Efstathiou, G., \& Jedrzejewski, R.I. 1984, Adv. Space Res., 3, 379

Efstathiou G., Sutherland W.J., Maddox S.J., 1990, Nat, 348, 705

Fry J.N., 1984, ApJ, 279, 499

Gaztanaga E., 1994, MNRAS, 268, 913

Gaztanaga E., Croft R.A.C., Dalton G.B., 1995, MNRAS, 276, 336

Gaztanaga E., Frieman J.A., 1994, ApJ, 437, L13

Jing, Y.P. 1997, in preparation

Jing Y.P., Zhang J.L., 1989, ApJ, 342, 639

Jing, Y.P., Börner, G. 1997, A\&A, 318, 667

Jing, Y.P., Börner, G. 1997, in preparation

Matarrese, S., Verde, L., Heavens, A. 1997, MNRAS (in press); astro-ph/9706059

Matsubara T., Suto Y., 1994, ApJ, 420, 497

Mo H.J., White S.D.M., 1996, MNRAS, 282, 347

Mo, H.J., Jing, Y.P., White, S.D.M., 1997, MNRAS, 284, 189

Peebles P.J.E., 1980, The Large-Scale Structure of the Universe,Princeton University Press, Princeton

Suto, Y., 1993, Prog. Theor. Phys. 90, 1173 